\title{
Inferring superposition and entanglement in evolving systems from measurements in a single basis
}

\author{
Bella Schelpe, Adrian Kent*, William Munro and Tim Spiller \\ Hewlett-Packard Laboratories, Filton Road, Stoke Gifford, Bristol BS34 8QZ, U.K.
}

(Dated: March 2003 (revised))

\begin{abstract}
We discuss what can be inferred from measurements on evolving one and two qubit systems using a single measurement basis at various times. We show that, given reasonable physical assumptions, carrying out such measurements at quarter-period intervals is enough to demonstrate coherent oscillations of one or two qubits between the relevant measurement basis states. One can thus infer from such measurements alone that an approximately equal superposition of two measurement basis states has been created during a coherent oscillation experiment. Similarly, one can infer that a near maximally entangled state of two qubits has been created part way through an experiment involving a putative SWAP gate. These results apply even if the relevant quantum systems are only approximate qubits. We discuss applications to fundamental quantum physics experiments and quantum information processing investigations.
\end{abstract}

PACS numbers: 03.67-a,03.67.Lx,03.65.Ud,85.25.Dq,85.35.Gv

\section{INTRODUCTION}

It is well known from tomography that given many copies of a quantum state, measuring enough observables allows reconstruction of the state [1]. However, the relevant quantum tomographic schemes generally require measurements in more than one basis. In many systems this doesn't present any difficulties, for example polarisation measurements on photons are easily carried out in any basis. However for many condensed matter qubit systems, there is usually just one natural measurement basis, often defined to be the computational basis or, in spin- $1 / 2$ notation, the $z$-basis: $|0\rangle=\left|\uparrow_{z}\right\rangle$ and $|1\rangle=\left|\downarrow_{z}\right\rangle$. This is the motivation for this paper. We would like to know what, if anything, can be inferred about the evolution of a one or two qubit system if measurements on each qubit are restricted to a single basis, but can be made at different times. Specifically, we are interested in the problems of demonstrating coherent oscillations between $|0\rangle$ and $|1\rangle$ in an experimental arrangement that is meant to function as a quantum NOT gate, and between $|0\rangle|1\rangle$ and $|1\rangle|0\rangle$ in an arrangement that is meant to function as a quantum SWAP gate. Our goal is thus less ambitious than full quantum state tomography. However, the experimental simplicity of our approach could be used to infer evidence for the fundamental quantum phenomena of superposition and entanglement in new candidate qubit systems, which are currently not amenable to full tomography [2]. Other approaches such as "entanglement witnesses" have been proposed to test whether a system is entangled or now 3, 4]. These generally however require measurements outside the computational basis that we wish to restrict our attention too.

Consider first the NOT gate. The standard coherent oscillation scenario is ideally realised by arranging for the spin qubit Hamiltonian to be of the form $H=-\frac{\Gamma}{2} \sigma_{x}$, with $\hbar$ set to 1 . Inputting either computational basis state ( $\sigma_{z}$ eigenstate), the probabilities of finding the sys- tem in these basis states oscillate coherently with period $2 \pi \Gamma^{-1}$. With a dimensionless time $T \equiv \Gamma t$, a single oscillation is complete at $T=2 \pi$. A NOT gate is effected by the action of $H$ for a time $T=\pi$ and a root NOT by acting for $T=\pi / 2$.

The usual approach to inferring that a superposition $|0\rangle+|1\rangle$ really was created at $T=\pi / 2$ in an experiment is to plot out the sinusoidal oscillations of the computational basis measurement probabilities in detail. This is done by preparing, evolving for a small fraction of $T=2 \pi$, measuring in the computational basis, repeating many times to build up statistics of $p(0)$ and $p(1)$ for this value of $T$, then incrementing $T$ and repeating the whole procedure until one reaches $T=2 \pi$.

Coherent oscillation of the quantum state appears much the simplest and likeliest explanation of a sinusoidal oscillation in the measurement probabilities. One might worry, though, that some other evolutions could also be consistent with the data. For example, measurement probabilities $p(0)=p(1)=1 / 2$ at $\pi / 2$ would also be obtained if the system generated a mixed state $|0\rangle\langle 0|+| 1\rangle\langle 1|$, rather than the desired pure state $|0\rangle+|1\rangle$, at this point. Conversely, one might wonder whether carrying out measurements at many evolution times is necessary or even helpful for inferring the state at $\frac{\pi}{2}$.

A further issue in inferring superposition is that real world plots will at best only be approximately consistent with ideal coherent oscillation: for example, the experimental oscillation amplitudes will decay over time. Moreover, the relevant quantum system may only be an approximate qubit. Textbook discussions of quantum computing often assume the qubits are exact, in that they correspond to two-dimensional quantum systems such as the polarisation states of a single photon. However, real world qubits may be approximate, corresponding to systems which live in a larger Hilbert space but have parameters set so that two of the states have very small transition probabilities to any of the rest. One would 
ideally like to be able to estimate how close the state generated under these conditions is to the superposition obtained by an ideal evolution.

In this paper we show that, in fact, the existence of coherent superpositions can be inferred simply by carrying out computational basis measurements every quarterperiod, without a full sinusoidal oscillation plot. This is true for both the NOT and SWAP gate evolutions, and even for approximate qubits and imperfect evolutions. In all cases a reasonably simple bound on the fidelity to an ideal superposition state can be obtained. Our arguments require physically reasonable and fairly minimal assumptions, which are necessary to infer coherent superposition even if measurements are made at many different evolution times. Our procedure thus simplifies the experimenter's task considerably, while providing evidence for the results inferred.

The experimental motivation for considering approximate as well as exact qubits comes from condensed matter systems where the qubits are often not exact. Two interesting condensed matter approximate qubits are based on superconductivity. The computational basis states can either be two charge states of a microscopic superconducting island (or box) differing by a single Cooper pair 5], or two flux states of a closed superconducting ring $[6$, ]].

Such systems are particularly interesting because they form one of a number of promising routes towards realising condensed matter qubits for quantum computing. Coherent oscillations have already been seen with an approximate charge qubit [8], similar results have been seen with related charge-phase systems [9, 10, 11] and very recently two charge qubits have been coupled [12], leading to the possibility of entanglement. Flux systems may also be useful qubits and, in addition, these are very interesting from the fundamental quantum physics viewpoint. Two flux states of a superconducting ring, differing by the flux quantum $\Phi_{0}=h / 2 e$, correspond to macroscopically distinct circulating currents in the ring, so a superposition of two such states would be a real analogue of Schrödinger's cat. Flux experiments [13, 14] have provided evidence for flux superpositions in the frequency domain, in effect through spectroscopy, although these experiments did not exhibit time domain oscillations. Various experiments to demonstrate coherent flux oscillations are underway, and the first results are emerging [15]. Flux or current oscillations will be recognised as clear evidence for Schrödinger cat superpositions (in the same way that charge oscillations [8] have been recognised as showing clear evidence for charge superpositions). The single approximate qubit results we present here are particularly relevant for the ongoing flux oscillation experiments.

For two qubit systems there are a number of potential gates that can be investigated. Here however we restrict our attention to the SWAP and root SWAP gates. These are useful gates to consider, because as is well known, universal quantum computations can be carried out by com- bining root SWAP with single qubit gates. A swapping evolution (involving just two of the four basis states of a two qubit system) is a natural one for various superconducting systems [12, 16, 17]. For a system of two spins, $a$ and $b$, a swapping evolution can be realised with an interaction Hamiltonian of the Heisenberg form $H=\frac{J}{4} \underline{\sigma}_{a} \cdot \underline{\sigma}_{b}$. This interaction is relevant for electron spins in coupled quantum dots [18] and other spin realisations of qubits in condensed matter systems. The computational basis states $|0\rangle|1\rangle$ and $|1\rangle|0\rangle$ swap after a dimensionless time $J t \equiv T=\pi$. After a time $T=\pi / 2$ a root SWAP gate is effected [18]. Approximate implementations of root SWAP have also been proposed for other condensed matter qubit systems, electron on helium [19] and Coulomb coupled quantum dots [20] where the approximate qubits are fictitious spins, so the swapping evolution is relevant for numerous qubit realisations.

\section{PHYSICAL ASSUMPTIONS}

Our fundamental assumption is that, in any experiment we consider, we can characterize the Hilbert space corresponding to the quantum system(s) of interest, and distinguish system degrees of freedom from those corresponding to the rest of the experimental apparatus, thermal radiation, and the rest of the outside world, all of which we collectively refer to as the environment. So the total Hilbert space $\mathcal{H}_{\text {total }}$ can be factored as

$$
\mathcal{H}_{\text {total }}=\mathcal{H}_{S} \otimes \mathcal{H}_{E} \text { or } \mathcal{H}_{S_{1}} \otimes \mathcal{H}_{S_{2}} \otimes \mathcal{H}_{E}
$$

for a one or two qubit system respectively. Exact qubits correspond to two dimensional spaces $\mathcal{H}_{S}$ and $\mathcal{H}_{S_{i}}$ with an orthonormal basis $|0\rangle,|1\rangle$. For approximate qubits we can without loss of generality consider countably infinite dimensional spaces with a basis $|0\rangle,|1\rangle,|2\rangle, \ldots$, with the two distinguished states $|0\rangle$ and $|1\rangle$ defining the qubit computational basis.

We assume that the system-environment interactions are such that we can initialize the system in any computational basis state without materially affecting the environment and can treat the environment as effectively constant during subsequent evolutions. More precisely:

(i) the state of the experiment at any time can be characterized by a density matrix $\rho_{S}$ or $\rho_{S_{1} \otimes S_{2}}$ describing (only) the system state.

(ii) the evolution of the system between any times $t$ and $t^{\prime}>t$ is described by a quantum operation $\mathcal{E}_{t, t^{\prime}}$ which is trace-preserving, convex linear on density matrices and completely positive.

(iii) the operations $\mathcal{E}_{t, t^{\prime}}$ is independent of the initial state of the system and is the same in each experimental run.

We turn now to the detailed analyzes of the various qubit evolutions and what may be inferred about superposition and entanglement from measurements. 


\section{AN EXACT QUBIT}

There is a particularly simple strategy for inferring superpositions in the case of an experiment testing a prototype NOT gate by attempting to produce coherent oscillations of a single exact qubit. We run the experiment setting the initial system state to be either of the two computational basis states $\rho_{0}=|0\rangle\langle 0|$ and $\rho_{1}=|1\rangle\langle 1|$. Assume that we have identified, either theoretically or empirically, a time $t$ such that $\mathcal{E}_{t} \equiv \mathcal{E}_{0, t}$ implements an approximate NOT operation. We can quantify the degree of approximation by carrying out a series of experiments in which computational basis measurements are performed at time $t$ and estimating the probabilities of obtaining 0 and 1 from the states evolved from $\rho_{1}$ and $\rho_{0}$. We write

$$
p_{i}^{j}=\left\langle i\left|\mathcal{E}_{t}\left(\rho_{j}\right)\right| i\right\rangle
$$

To proceed we need the following definitions and results 21]. The trace distance between states $\rho$ and $\rho^{\prime}$ is defined as $D\left(\rho, \rho^{\prime}\right)=\frac{1}{2} \operatorname{Tr}\left|\rho-\rho^{\prime}\right|$, where $|A|=\left(A^{\dagger} A\right)^{\frac{1}{2}}$ is the positive square root of $A^{\dagger} A$. It has the following properties. First, $D$ is a metric: $D\left(\rho, \rho^{\prime}\right)=0$ if and only if $\rho=\rho^{\prime}, D\left(\rho, \rho^{\prime}\right)=D\left(\rho^{\prime}, \rho\right)$ and $D\left(\rho, \rho^{\prime \prime}\right) \leq$ $D\left(\rho, \rho^{\prime}\right)+D\left(\rho^{\prime}, \rho^{\prime \prime}\right)$. Second, $D$ is non-increasing under trace-preserving quantum operations: $D\left(\mathcal{E}(\rho), \mathcal{E}\left(\rho^{\prime}\right)\right) \leq$ $D\left(\rho, \rho^{\prime}\right)$. Third, the fidelity $F\left(\rho, \rho^{\prime}\right)=\operatorname{Tr}\left(\rho^{\frac{1}{2}} \rho^{\prime} \rho^{\frac{1}{2}}\right)$ and $\bar{D}$ obey

$$
1-F^{2}\left(\rho, \rho^{\prime}\right) \leq D\left(\rho, \rho^{\prime}\right) \leq\left(1-F\left(\rho, \rho^{\prime}\right)\right)^{\frac{1}{2}} .
$$

When $\rho$ is a pure state we have a stronger lower bound:

$$
1-F\left(\rho, \rho^{\prime}\right) \leq D\left(\rho, \rho^{\prime}\right) .
$$

We will need a further result which is that when $\rho$ is a given pure state, $F\left(\rho, \rho^{\prime}\right)$ is the same for all $\rho^{\prime}$ lying on a disc orthogonal to the radius joining the centre of the Bloch sphere to $\rho$.

This can be seen by considering $\rho=|0\rangle\langle 0|$. The pure states on a given disc are $\cos \frac{\theta}{2}|0\rangle+e^{i \phi} \sin \frac{\theta}{2}|1\rangle$. The fidelities of all these states to $|0\rangle\langle 0|$ are $\cos ^{2} \frac{\theta}{2}$. Since all mixed states on the disc are mixtures of these pure states and fidelity between a mixed and pure state is linear in the mixed state, all states on the disc have fidelity $\cos \frac{\theta}{2}$ to $|0\rangle$.

If our system really is an exact qubit, we find that $p_{0}^{1}+p_{1}^{1}=1=p_{0}^{0}+p_{1}^{0}$. Geometrically, $\mathcal{E}_{t}\left(\rho_{1}\right)$ lies in the intersection of the Bloch sphere with a plane at height $2 p_{0}^{1}-1$ above the equator and parallel to it, while $\mathcal{E}_{t}\left(\rho_{0}\right)$ lies in the intersection of the sphere with a parallel plane
$2 p_{1}^{0}-1$ below the equator. Since the trace distance is half the Euclidean distance within the Bloch sphere, we have that

$$
D\left(\mathcal{E}_{t}\left(\rho_{1}\right), \mathcal{E}_{t}\left(\rho_{0}\right)\right) \geq\left(p_{0}^{1}+p_{1}^{0}-1\right)
$$

As the trace distance is non-increasing during quantum evolution, the two qubits evolved from $\rho_{0}$ and $\rho_{1}$ must always have been separated by at least this distance before time $t$. We can picture the qubit evolution as defining that of a perhaps contracting rod (whose endpoints are the qubits) moving inside the Bloch sphere. At some time before $t$ during the evolution, this 'rod' must be parallel to the equatorial plane. The condition (4) defines a cylinder of Euclidean radius $p_{0}^{1}+p_{1}^{0}-1$ outside which at least one of the qubits must lie at this time. The state most distant from any pure state on the equator must therefore enter a volume bounded by the Bloch sphere and a disc normal to the radius to some equatorial point and subtended by a geometric angle defined by $\cos \theta=p_{0}^{1}+p_{1}^{0}-1$. Using equation (3) and the above result that all $\rho^{\prime}$ lying on a disc orthogonal to the relevant radius will have the same fidelity, we can see that the fidelity of this qubit to a maximally superposed state of the form $|0\rangle+e^{i \phi}|1\rangle$ must be no less than $\left(\frac{p_{0}^{1}+p_{1}^{0}}{2}\right)$. Hence we can infer the creation of a near-maximally superposed state during the experiment - though note that this argument does not identify which superposed state was approximated, or when.

\section{AN APPROXIMATE QUBIT}

Now suppose our system is only approximately characterized by a qubit. Clearly, any argument for coherent oscillations will need some measure of how closely the evolved state resembles a qubit. For any given state $\rho$ that can be repeatedly prepared, we can obtain a good measure as follows. Define $P$ to be the projection onto the two-dimensional qubit space, and note that if $p_{0}^{\rho}$ and $p_{1}^{\rho}$ are the probabilities of getting outcomes 0 and 1 when measuring in the computational basis, we can obtain $\operatorname{Tr}(P \rho)=p_{0}^{\rho}+p_{1}^{\rho}$. Let $\rho_{P}=P \rho P / \operatorname{Tr}(\rho P)$ be the normalized density matrix given by projecting $\rho$ into the qubit space. Then $F\left(\rho_{P}, \rho\right)=(\operatorname{Tr}(P \rho))=\left(p_{0}^{\rho}+p_{1}^{\rho}\right)$ and so we have

$$
D\left(\rho_{P}, \rho\right) \leq\left(1-p_{0}^{\rho}-p_{1}^{\rho}\right)^{\frac{1}{2}}
$$

We can now proceed as previously and obtain estimates for the probabilities (1) associated with an approximate NOT evolution, giving us that

$$
\begin{aligned}
D\left(\mathcal{E}_{t}\left(\rho_{1}\right), \mathcal{E}_{t}\left(\rho_{0}\right)\right) & \geq D\left(\mathcal{E}_{t}\left(\rho_{1}\right)_{P}, \mathcal{E}_{t}\left(\rho_{0}\right)_{P}\right)-D\left(\mathcal{E}_{t}\left(\rho_{0}\right), \mathcal{E}_{t}\left(\rho_{0}\right)_{P}\right)-D\left(\mathcal{E}_{t}\left(\rho_{1}\right), \mathcal{E}_{t}\left(\rho_{1}\right)_{P}\right) \\
& \geq\left(q_{0}^{1}+q_{1}^{0}-1\right)-\left(1-p_{0}^{0}-p_{1}^{0}\right)^{\frac{1}{2}}-\left(1-p_{0}^{1}-p_{1}^{1}\right)^{\frac{1}{2}}
\end{aligned}
$$


where $q_{0}^{1}=p_{0}^{1} /\left(p_{0}^{1}+p_{1}^{1}\right)$ and $q_{1}^{0}=p_{1}^{0} /\left(p_{0}^{0}+p_{1}^{0}\right)$.

To complete the argument, we need some way of bounding the possible deviation from an exact qubit at a point when we expect a near-maximally superposed state to have been created. A general bound of the form $\operatorname{Tr}\left(P \mathcal{E}_{t^{\prime}}\left(\rho_{i}\right)\right) \geq 1-\delta$ for all times $t^{\prime}<t$ and for $i=0,1$ would suffice. However, getting good empirical evidence for such a bound would require carrying out a series of measurements at many times between 0 and $t$, which would require the same amount of experimental labour as plotting sinusoidal oscillations, and would still leave the worry that the deviation might have been greater at some unmeasured intervening point.

A more watertight procedure is to identify empirically a time $t_{1 / 2}<t$ (for coherent oscillations we expect $t_{1 / 2} \approx$ $t / 2)$ such that, if we write $r_{i}^{j}=\left\langle i\left|\mathcal{E}_{t_{1 / 2}}\left(\rho_{j}\right)\right| i\right\rangle$, we have $r_{0}^{0}, r_{1}^{0}, r_{0}^{1}, r_{1}^{1} \approx 1 / 2$. We can then directly argue that the evolution came close to a maximally superposed state at $t_{1 / 2}$ as follows.

Writing $\sigma_{i}=\mathcal{E}_{t_{1 / 2}}\left(\rho_{i}\right)$ and $\sigma_{i}^{P}=P \sigma_{i} P / \operatorname{Tr}\left(\sigma_{i} P\right)$ for $i=0,1$ we have $F\left(\sigma_{i}^{P}, \sigma_{i}\right)=\left(\operatorname{Tr}\left(P \sigma_{i}\right)\right)=\left(r_{0}^{i}+r_{1}^{i}\right)$ and

$$
\begin{aligned}
D\left(\sigma_{0}^{P}, \sigma_{1}^{P}\right) & \geq D\left(\sigma_{0}, \sigma_{1}\right)-D\left(\sigma_{0}, \sigma_{0}^{P}\right)-D\left(\sigma_{1}, \sigma_{1}^{P}\right) \\
& \geq\left(q_{0}^{1}+q_{1}^{0}-1\right)-\left(1-p_{0}^{0}-p_{1}^{0}\right)^{\frac{1}{2}}-\left(1-p_{0}^{1}-p_{1}^{1}\right)^{\frac{1}{2}}-\left(1-r_{0}^{0}-r_{1}^{0}\right)^{\frac{1}{2}}-\left(1-r_{0}^{1}-r_{1}^{1}\right)^{\frac{1}{2}} .
\end{aligned}
$$

We will write the right hand side of this last inequality as $1-\epsilon^{\prime}$ and let $s_{j}^{i}=r_{j}^{i} /\left(r_{0}^{i}+r_{1}^{i}\right)$, with $\Delta s_{j}^{i}=s_{j}^{i}-1 / 2$. A little Bloch sphere trigonometry shows that at least one of the states $\sigma_{i}^{P}$ must lie in a cap, centred on a maximally superposed state, whose Euclidean height is no more than

$$
\delta=1-\sqrt{\left(1-\epsilon^{\prime}\right)^{2}-\left(\Delta s_{0}^{1}+\Delta s_{1}^{1}\right)^{2}} .
$$

The relevant $\sigma_{i}^{P}$ is separated from the maximally superposed state $\left|\psi_{M}\right\rangle$ by trace distance no more than $\left(\frac{\delta^{2}}{4}+\left(\Delta s_{0}^{i}\right)^{2}\right)^{\frac{1}{2}}$, and thus we have

$$
\begin{aligned}
D\left(\sigma_{i},\left|\psi_{M}\right\rangle\right) & \leq D\left(\sigma_{i}, \sigma_{i}^{P}\right)+D\left(\sigma_{i}^{P},\left|\psi_{M}\right\rangle\right) \\
& \leq\left(1-r_{0}^{i}-r_{1}^{i}\right)^{\frac{1}{2}}+\left(\frac{\delta^{2}}{4}+\left(\Delta s_{0}^{i}\right)^{2}\right)^{\frac{1}{2}} \\
& \leq \max _{j}\left\{\left(1-r_{0}^{j}-r_{1}^{j}\right)^{\frac{1}{2}}+\left(\frac{\delta^{2}}{4}+\left(\Delta s_{0}^{j}\right)^{2}\right)^{\frac{1}{2}}\right\} .
\end{aligned}
$$

This last expression is experimentally measurable and bounds the separation between one of the evolved states and a maximally superposed state, although the argument does not identify either state.

\section{ENTANGLEMENT GENERATION VIA CANDIDATE SWAP GATES}

Consider now an experiment implementing a candidate SWAP gate on a system of two exact or approximate qubits. If we initialize the system in the state $|0\rangle|1\rangle$, an exact exchange interaction would evolve the system to $|1\rangle|0\rangle$ via a maximally entangled state of the form $\left|\psi_{M}\right\rangle=\frac{1}{\sqrt{2}}\left(|0\rangle|1\rangle+e^{i \phi}|1\rangle|0\rangle\right)$. We can consider the two qubits as defining a single effective qubit in the two dimensional space with computational basis $|0\rangle|1\rangle$ and $|1\rangle|0\rangle$. If the system state is initially in this space, it will, to good approximation, remain there provided that the evolution is well approximated by an exchange interaction. Our results for the NOT evolution of approximate qubits thus apply directly.

Moreover, by carrying out appropriate measurements, we can verify that an entangled state is generated during the candidate SWAP evolution and give figures of merit for the entanglement. Define the projection $Q$ to be on the four dimensional space defined by the two qubits. A measurement of $Q$ on the state $\sigma_{i}$ would produce the exact qubit state $\sigma_{i}^{Q}=Q \sigma_{i} Q / \operatorname{Tr}\left(Q \sigma_{i}\right)$ with probability $p_{Q}=\sum_{l=0,1 ; m=0,1} p_{l m}^{i}$, where $p_{l m}^{i}=\left\langle l\left|\left\langle m\left|\sigma_{i}\right| l\right\rangle\right| m\right\rangle$. For any maximally entangled state $\left|\psi_{M}\right\rangle$ we have

$$
\begin{aligned}
\max _{i}\left\langle\psi_{M}\left|\sigma_{i}^{Q}\right| \psi_{M}\right\rangle \geq & \min _{j}\left\{\frac{p_{01}^{j}+p_{10}^{j}}{p_{00}^{j}+p_{10}^{j}+p_{01}^{j}+p_{11}^{j}}\right\} \times \\
& \max _{k}\left\{\left\langle\psi_{M}\left|\sigma_{k}^{P}\right| \psi_{M}\right\rangle\right\} .
\end{aligned}
$$

As we can calculate both expressions on the right hand side, we have a lower bound on the fidelity of a $\sigma_{i}^{Q}$ to a maximally entangled state. If this is greater than one half, the relevant $\sigma_{i}^{Q}$ is entangled, and as $\sigma_{i}^{Q}$ can be obtained from $\sigma_{i}$ by local measurements, $\sigma_{i}$ is also entangled. The advantage of this procedure over more efficient ways of measuring entanglement 3 , 4, 22, 23] is that it requires no gates other than the one being tested.

\section{DISCUSSION}

We have shown that, if a candidate NOT gate acts on an exact qubit, the qubit must pass close to an equal weight superposition of $|0\rangle$ and $|1\rangle$ if the NOT operation is demonstrated to work accurately on both basis states as inputs. For an approximate qubit measurements are also needed half way through the NOT operation, to check that the system has not wandered far out of the qubit subspace. However, in both cases superposition can 
be inferred without plotting oscillations in detail, given reasonable physical assumptions.

These physical assumptions should hold true in the types of experiment we consider. However, it should be stressed that they could in principle be violated given sufficient pathologies. To take an extreme example, one could imagine a single qubit experiment in which, unknown to the experimenter, the environment happened to contain a second qubit, coupled to the first by a fast but only infrequently acting swap gate. The initially prepared computational basis state in the first qubit could then be exchanged with a maximally mixed state in the second, rotated via a fortuitous NOT operation in the second qubit, and then exchanged back into the first. Applying our inequalities would lead to the incorrect conclusion that the first qubit evolves through a near-maximal superposition state. In fact, in this case, such a state is indeed created, but in the second qubit lurking in the environment. Of course, it is very unlikely indeed that any experiment will accidentally incorporate a hidden qubit or anything similar.

The arguments used to infer a superposition in a single qubit from computational basis measurements can also be used to infer entanglement in certain multi-qubit systems. For a two qubit system arranged to effect a SWAP operation, demonstration that this works well for the two inputs $|0\rangle|1\rangle$ and $|1\rangle|0\rangle$ and measurements at the half way point (root SWAP) showing that the system hasn't wandered far out of the subspace spanned by these states are sufficient to infer that the system passed close to a maximally entangled state. Fidelities can be bounded and the existence of entanglement established, even for states well away from being maximally entangled. Once again, all this works whether the qubits are exact or approximate.

Clearly, for candidate condensed matter systems to be feasible qubits for quantum computation, reliable single qubit gates will eventually need to be constructed. This would remove the need to restrict to measurements in the computational basis, and would make tomography much simpler. However, state of the art is currently well short of this goal. At present, experimenters are trying to realise approximate one and two condensed matter qubit gates and test them. In particular, recent superconducting experiments [12] show that the coupling of two condensed matter qubits and investigations of entanglement are now becoming possible. We believe that the results presented here should be extremely useful during the present investigative period. Our technique achieves less than full quantum state tomography would, but requires considerably less experimental effort, while providing a simple way of inferring essential quantum behaviour in new candidate qubit experiments. Even in the longer term, our results should be relevant for fundamental investigations such as creating multi-qubit GHZ states or entangling two Schrödinger cats, which may well be carried out on systems where our measurement restrictions apply.

\section{Acknowledgements}

This work was supported in part by the European project EQUIP, IST-1999-11053. We thank Simon Benjamin, Lucien Hardy, Brendon Lovett and Martin Plenio for valuable discussions.
[*] Current Address: DAMTP, Centre for Mathematical Sciences, University of Cambridge, Wilberforce Road, Cambridge, CB3 OWA, U.K.

[1] D. F. V. James et al., Phys. Rev. A 64, 052312 (2001); R. T. Thew et al., Phys. Rev. A 66, 012303 (2002); $\mathrm{U}$. Leonhardt, Measuring the Quantum state of Light, (Cambridge University Press, 1997).

[2] Collections of papers on qubit realisations can be found in: S. Braunstein and H.-K. Lo (eds.) Fortschr. Phys. 48 (9-11) (2000); R. G. Clark (ed.), Experimental Implementation of Quantum Computation, (Rinton Press, 2001).

[3] M. Lewenstein et al., Phys. Rev. A 62, 052310 (2002)

[4] O. Guhne et al., Phys. Rev. A 66, 062305 (2002)

[5] A. Shnirman et al., Phys. Rev. Lett. 79, 2371 (1997).

[6] M. F. Bocko et al., IEEE Trans. on Appl. Superconductivity 7, 3638 (1997).

[7] J. E. Mooji et al., Science 285, 1036 (1999).

[8] Y. Nakamura et al., Nature 398, 786 (1999).

[9] D. Vion et al., Science 296, 886 (2002).

[10] Y. Yu et al., Science 296, 889 (2002).
[11] J. M. Martinis et al. Phys. Rev. Lett. 89, 117901 (2002).

[12] Yu. A. Pashkin et al. Nature 421, 823 (2003).

[13] J. R. Friedman et al., Nature 406, 43 (2000).

[14] C. H. van der Wal et al., Science 290, 773 (2000).

[15] I. Chiorescu et al., Sciencexpress 1081045 (2003).

[16] F. W. Strauch et al., quant-ph/0303002

[17] The charge-phase qubit of ref. 7 can be biased so the measurable computational basis states are energy eigenstates (charge superpositions). The Coulomb interaction between two such qubits would provide a swapping interaction between $|0\rangle|1\rangle$ and $|1\rangle|0\rangle$.

[18] G. Burkard et al., Phys. Rev. B60, 11404 (1999).

[19] M. J. Lea et al., Fortschr. Phys. 48, 1109 (2000).

[20] J. H. Jefferson et al., Phys. Rev. A 66, 042328 (2002).

[21] See M. A. Nielsen and I. L. Chuang, Quantum Computation and Quantum Information (Cambridge University Press, 2000), section 9.2.

[22] P. Horodecki and A. Ekert, Phys. Rev. Lett. 89, 127902 (2002); P. Horodecki, quant-ph/0111082

[23] J. Fiurasek, Phys. Rev. A 66, 052315 (2002). 